\title{
Elaboración de una bebida pasteurizada a partir de un extracto proteico de quinua (Chenopodium quinoa Willd.)
}

\section{(Elaboration of a pasteurized beverage from a quinoa (Chenopodium quinoa Willd.) protein extract)}

\begin{abstract}
Andrea Guallasamín-Dávila ${ }^{1}$, Jenny Ávila-Vélez ${ }^{1}$, Cristina Sotomayor-Grijalva ${ }^{1}$
Resumen:

La investigación consistió en evaluar parámetros para obtener una bebida de quinua. Los parámetros considerados fueron extracción de proteína soluble (PS) de quinua y dos condiciones de proceso (adición de estabilizante y temperatura de pasteurización). La concentración de PS en el extracto proteico se estudió a través de los factores: relación harina-solución de $\mathrm{NaOH}\left(1: 2.50,1: 3.25\right.$ y 1:4.00), temperatura (20 y $\left.45^{\circ} \mathrm{C}\right)$ y $\mathrm{pH}$ de acondicionamiento (7.50 y 8.00$)$, durante $30 \mathrm{~min}$ a escala de laboratorio. El extracto con mayor concentración de PS fue replicado a escala piloto y formulado con azúcar blanca, saborizante de vainilla y estabilizante $(0.00$ y $0.25 \%)$ para obtener la bebida, luego se evaluó la temperatura de pasteurización (120 y $130 \stackrel{\circ}{\circ}$ ). Las variables dependientes medidas al día 0 en la bebida fueron: variación de proteína soluble (VPS $\left.{ }_{b}\right)$, porcentaje en suspensión y reducción de la población microbiana (RPM). EI extracto obtenido en proporción 1:3.25 harina-solución de $\mathrm{NaOH}, 20{ }^{\circ} \mathrm{C}$ y pH 7.50 obtuvo $77.10 \pm 1.76 \%$ de PS. El tratamiento a $120 \stackrel{\circ}{ } \mathrm{C}$ con estabilizante $(0.25 \%)$ tuvo $2.36 \pm 0.25 \%$ de VPS y $98.33 \pm 1.53 \%$ en porcentaje en suspensión. La bebida obtenida alcanzó $2.36 \%$ de proteína.
\end{abstract}

Palabras clave: bebida vegetal; quinua; solubilización proteica; tratamiento térmico.

\begin{abstract}
:
The research consisted on evaluating parameters to obtain a quinoa beverage. Quinoa soluble protein (SP) extraction and two process conditions (addition of stabilizer and pasteurization temperature) were considered as parameters. The concentration of SP in the protein extract was studied through the factors: flour: $\mathrm{NaOH}$ solution ratio $(1: 2.50$, $1: 3.25$ and 1:4.00), conditioning temperature (20 and $\left.45{ }^{\circ} \mathrm{C}\right)$, and $\mathrm{pH}(7.50$ and 8.00$)$, for $30 \mathrm{~min}$ at laboratory scale. The protein extract with the highest SP concentration was replicated at pilot scale, and white sugar, vanilla flavor and stabilizer (0.00 and $0.25 \%)$ were added to formulate beverage. Then, the pasteurization temperature (120 and 135 $\left.{ }^{\circ} \mathrm{C}\right)$ was evaluated. The independent variables: soluble protein variation $\left(\mathrm{SPV}_{\mathrm{b}}\right)$, suspension percentage and microbial population reduction (MPR) were measured at day 0 . The protein extract that was obtained with a flour- $\mathrm{NaOH}$ solution ratio of 1:3.25, $20{ }^{\circ} \mathrm{C}$ and $\mathrm{pH} 7.5$ reached $77.10 \pm 1.76 \%$. The beverage had a VPS $-2.36 \pm 0.25 \%$, a suspension percentage of $98.33 \pm 1.53 \%$ and $2.36 \%$ of protein at $120 \stackrel{\circ}{\circ}$ with stabilizer $0.25 \%$.
\end{abstract}

Keywords: plant-based milk substitutes; quinoa; protein solubilization; heat treatment.

\footnotetext{
${ }^{1}$ Escuela Politécnica Nacional, Quito, Ecuador ( \{andrea.guallasamin, jenny.avila, cristina.sotomayor\} @epn.edu.ec )
} 


\section{Introducción}

Las bebidas vegetales son suspensiones o emulsiones de legumbres, frutos secos, cereales y pseudocereales que han mantenido un margen de crecimiento en el mercado debido a sus propiedades funcionales (Mäkinen et al., 2015; Ismail, 2015). En un inicio fueron concebidas como productos alternativos para personas alérgicas a la proteína animal o intolerantes a la lactosa (Mäkinen et al., 2015). Sin embargo, hoy en día, la tendencia global hacia un estilo de vida más saludable ha provocado que más consumidores incluyan este producto en su dieta diaria por su naturaleza nutritiva, bajo contenido calórico, fácil digestión, entre otros beneficios (Soteras, 2011).

Bajo esta perspectiva, las industrias de bebidas buscan vegetales, con mejores potenciales de industrialización y bajos costos de producción; la quinua (Chenopodium quinoa Willd.), por ejemplo, cumple con este perfil (FAO, 2011). La quinua es un pseudocereal nativo de los Andes reconocido por su contenido y calidad proteica (10.4 $17 \%$ ) (Reyes-Montaño et al., 2006). Es fuente de aminoácidos esenciales que pueden satisfacer los patrones de nutrición humana (Bergesse et al., 2015), entre ellos se destacan la lisina, importante para el desarrollo físico e intelectual y otros azufrados importantes que no se encuentran en los cereales (Oficina Comercial de Ecuador \& Dirección de Inteligencia Comercial e Inversiones, 2011). Su balance de aminoácidos esenciales es similar a la caseína, proteína de la leche (Jacobsen et al., 2003). Además, es libre de gluten, colesterol y presenta baja alergenicidad (Chito et al., 2017; Villacrés et al., 2011).

Estados Unidos, Francia, Noruega y Alemania, entre otros países, han incursionado en el área de bebidas a partir de quinua; no obstante en Ecuador, uno de los mayores productores, el procesamiento aún se concentra en el grano escarificado para exportación y pocos derivados agroindustriales son producidos. Es así que se plantea la elaboración de una bebida a base de quinua variedad Tunkahuan por ser la de mayor producción a nivel nacional, de sabor "dulce" (bajo contenido de saponina) y no utilizar alta cantidad de bioinsumos (compost, bioles, etc) (Oficina Comercial de Ecuador \& Dirección de Inteligencia Comercial e Inversiones, 2011; Pro Ecuador, 2015).

Las operaciones de producción a gran escala son limitadas para bebidas de quinua (Mäkinen et al. 2015). Los estudios acerca de la elaboración se enfocan en incrementar el contenido final de proteína a través de procesos como germinación y malteado del grano o hidrólisis enzimática previo al tratamiento térmico tipo HTST (altas temperaturas en cortos tiempos, donde la proteína se ve afectada por el calor) (Kaur \& Tanwar, 2016; Pineli et al. 2015); por lo demás la literatura científica es escasa (Sethi et al., 2016).

Según Zayas (1997), la industria de bebidas prioriza el uso de proteínas solubles porque ayudan a mantener un sistema coloidal. Los factores externos que afectan a la solubilidad proteica son: $\mathrm{pH}$, temperatura, composición de la solución y fuerza iónica (Trevino et al., 2008). En esta investigación, se estudiará la influencia de los tres primeros factores con el fin de obtener un extracto proteico base en la preparación de la bebida. Además, publicaciones revelan que la pasteurización de bebidas vegetales a altas temperaturas no afecta negativamente a la proteína y favorece la retención de nutrientes con mínimas alteraciones químicas (Kwok \& Niranjan, 1995). Por tanto, se planteó evaluar el efecto de altas temperaturas de pasteurización (120 y $135^{\circ} \mathrm{C}$ ), en bebidas con $(0.25 \%)$ y sin estabilizante $(0.00 \%)$, sobre la concentración de proteína soluble (PS), estabilidad física y calidad microbiológica.

\section{Materiales y metodología}

Los resultados de este estudio fueron recopilados en las instalaciones del Laboratorio de Bioprocesos y Planta Piloto del Departamento de Ciencias de Alimentos y Biotecnología de la Facultad de Ingeniería Química y Agroindustria de la Escuela Politécnica Nacional. 


\subsection{Materiales}

Los materiales utilizados durante el desarrollo de la investigación se encuentran detallados en la Tabla 1.

Tabla 1. Descripción de materiales

\begin{tabular}{|l|l|l|}
\hline Material & Características/Descripción & $\begin{array}{l}\text { Casa comercial / } \\
\text { Fabricante }\end{array}$ \\
\hline Granos de quinua & $\begin{array}{l}- \text { Desaponificados } \\
- \text { Variedad Tunkahuan }\end{array}$ & Cereales Andinos \\
\hline Hidróxido de sodio & $\begin{array}{l}- \text { Grado analítico } \\
-99 \% \text { pureza }\end{array}$ & Merck \\
\hline Ácido clorhídrico & $\begin{array}{l}- \text { Grado técnico } \\
-35 \% \text { pureza }\end{array}$ & J. T. Baker \\
\hline Azúcar blanco & --- Corporación Favorita S.A \\
\hline Saborizante de vainilla & --- & Cramer S.A. \\
\hline Estabilizante Obsigel G50 & --- & Obsidián Cia Ltda. \\
\hline Placas petrifilm & $\begin{array}{l}- \text { Para recuento de bacterias } \\
\text { aerobias totales } \\
- \text { Para recuento de E. } \\
\text { coli/coliformes } \\
- \text { Para recuento de mohos y } \\
\text { levaduras }\end{array}$ & \\
\hline Botellas PET & -220 mL & 3 M \\
\hline
\end{tabular}

\subsection{Obtención y caracterización de la harina de quinua}

Los granos de quinua fueron procesados en un molino de pines (ALPINE, UPZ 160, Alemania), para obtener harina $y$, se realizaron análisis granulométrico, proximal y microbiológico. El análisis granulométrico se efectuó acorde con la norma ecuatoriana INEN 517 mediante una serie de tamices ASTM de mallas 70, 80,100, 200 y 230 ubicados en una máquina vibradora (W.S TYLER, RX-29, USA). El análisis proximal incluyó porcentaje de humedad (AOAC 925.10), proteína (AOAC 2001.11), extracto etéreo (AOAC 920.85), cenizas (AOAC 923.03), fibra cruda (ICC\#113) y carbohidratos totales por diferencia (AOAC, 2005; ICC, 1972). En el análisis microbiológico se evaluó la presencia de bacterias aerobias totales, coliformes, E. coli, mohos y levaduras mediante placas petrifilm. Para la preparación de la muestra, siembra (por duplicado), incubación e interpretación de resultados se utilizó la Guía de Interpretación 3M Petrifilm (3M, 2003). El tiempo y temperatura de incubación empleados se especifican en la Tabla 2.

Tabla 2. Tiempo y temperatura de incubación para distintos microorganismos

\begin{tabular}{|l|l|l|}
\hline Microorganismo & $\begin{array}{l}\text { Temperatura de incubación } \\
\text { (oC) }\end{array}$ & $\begin{array}{l}\text { Tiempo de Incubación } \\
\text { (horas) }\end{array}$ \\
\hline Bacterias aerobias totales & 30 & $72 \pm 1$ \\
\hline Coliformes & 35 & $24 \pm 2$ \\
\hline E. coli & 35 & $48 \pm 2$ \\
\hline Mohos y Levaduras & 20 & $72 \pm 1$ \\
\hline
\end{tabular}

\subsection{Determinación de las condiciones de solubilización de proteína de quinua a escala laboratorio}

El primer paso en la elaboración de la bebida de quinua fue la solubilización de proteína en un medio acuoso, esta etapa fue estudiada a nivel de laboratorio mediante un diseño experimental de tipo $3 \times 2 \times 2(n=3)$ donde se evaluaron los factores: relación harina-solución de $\mathrm{NaOH}$ (w: w) (1:2.50, 1:3.25 y 1:4.00), temperatura de acondicionamiento del extracto proteico (20 y $45^{\circ} \mathrm{O}$ ), y $\mathrm{pH}$ de acondicionamiento del 
extracto proteico (7.50 y 8.00). La variable de respuesta fue la variación de proteína soluble en el extracto proteico (VPS e $_{\text {). }}$

Para la solubilización de proteína se empleó la metodología detallada en Föste et al. (2015) con modificaciones. La harina de quinua fue dispersada en una solución de $\mathrm{NaOH}$; para las relaciones 1:2.50, 1:3.25 y 1:4.00 se emplearon soluciones de concentraciones $0.045,0.038$ y $0.030 \mathrm{M}$, respectivamente. La mezcla se homogenizó en una plancha de agitación y calentamiento (Corning, PC-420, USA) durante $60 \mathrm{~min}$, luego se centrifugó a $2500 \mathrm{rpm}(525 \times \mathrm{g})$ por $20 \mathrm{~min}$ en un equipo (IEC, HN-SII, USA). Se recuperó el sobrenadante (extracto proteico) y con la ayuda de un lienzo se retiraron los restos de material sólido. El extracto proteico fue ajustado a la temperatura y $\mathrm{pH}$ de acondicionamiento (indicados en el diseño experimental) e inmediatamente llevado a agitación por $30 \mathrm{~min}$. En el transcurso de este tiempo, se tomaron alícuotas de $1 \mathrm{~mL}$ cada 10 min, a partir de 0 min, con el fin de medir la concentración de proteína soluble (PS) mediante el método de Biuret (Valencia et al., 2012).

Mäkinen (2014) explica que luego de la solubilización proteica es recomendable una etapa de neutralización, por lo cual el pH de la muestra se reguló con $\mathrm{HCL} 1 \mathrm{~N}$ y un pHmetro (WTW, pH 3210, Alemania) hasta alcanzar el pH de acondicionamiento. Los tratamientos a $45^{\circ} \mathrm{C}$ se realizaron en baños María acoplados.

La VPS $S_{e}$ se calculó con la Ecuación 1:

Donde:

$$
V P S_{e}=P S_{30 \mathrm{~min}}-P S_{0 \mathrm{~min}}
$$

$\mathrm{VPS}_{\mathrm{e}}=$ Variación de proteína soluble en el extracto proteico (\%)

$\mathrm{PS}_{30 \text { min }}=$ Concentración de PS al finalizar el tiempo de acondicionamiento (\%)

$\mathrm{PS}_{0 \mathrm{~min}}=$ Concentración de PS al iniciar el tiempo de acondicionamiento (\%)

Las concentraciones de PS en porcentaje (\%) fueron halladas a base del peso de la harina, densidad del extracto proteico y diluciones de la muestra antes de la lectura en el espectrofotómetro.

\subsection{Determinación de las condiciones de procesamiento de la bebida de quinua pasteurizada a escala piloto}

En el procesamiento de la bebida se replicaron a escala piloto las condiciones de laboratorio que permitieron obtener una mayor concentración PS en el extracto proteico. Para determinar el mejor tratamiento se planteó un diseño experimental $2^{2}(n=3)$, donde se estudiaron los factores: temperatura de pasteurización $\left(120\right.$ y $135^{\circ} \mathrm{C}$ ) y adición de estabilizante $(0.00$ y $0.25 \%)$. Las variables de respuesta en la bebida fueron: variación de proteína soluble (VPS $\mathrm{V}_{\mathrm{b}}$ ), porcentaje en suspensión y reducción de la población microbiana (RPM) al día 0.

Debido al cambio de escala, la mezcla harina-solución de $\mathrm{NaOH}$ fue homogenizada en una batidora (CRYPTO PEERLESS, EC30, Inglaterra) a velocidad 1. Para la centrifugación se utilizó un equipo IEC, K, USA a 2500 rpm. Después, el extracto proteico fue filtrado en un lienzo y ajustado a la temperatura y $\mathrm{pH}$ de acondicionamiento, como se describe en el acápite anterior. El tiempo de acondicionamiento se ajustó en función de los resultados a escala de laboratorio. Para obtener la bebida, se añadió al extracto proteico, $8.00 \%$ de azúcar blanca, saborizante de vainilla $(1 \mathrm{~g} / \mathrm{kg})$ y estabilizante $(0.00 \circ 0.25 \%)$. Todos los ingredientes se incorporaron mediante agitación manual constante de $15 \mathrm{~min}$. La bebida formulada se pasteurizó (120 o $135^{\circ} \mathrm{C}$ ) en un equipo UHT/HTST de tubos en miniatura (Armfield, FT74XTS, Inglaterra) con un tiempo de residencia de $1 \mathrm{~s}$. Se envasó el producto final con el método de llenado en caliente, recomendado por Hariyadi, 2013, en botellas PET de $220 \mathrm{~mL}$.

La Ecuación 2 permitió determinar la VPS

Donde:

$$
V P S_{b}=P S_{B P}-P S_{B N P}
$$

$\mathrm{VPS}_{\mathrm{b}}=$ Variación de proteína soluble en la bebida pasteurizada al día 0 (\%) 
$\mathrm{PS}_{\mathrm{BP}}=$ Concentración de PS en la bebida pasteurizada (\%)

$\mathrm{PS}_{\mathrm{BNP}}=$ Concentración de PS en la bebida no pasteurizada (\%)

Para el cálculo se siguió el procedimiento expuesto en el acápite 2.3.

El porcentaje en suspensión en la bebida pasteurizada se evaluó a través del método sugerido por Pereira (2011), que consiste en colocar en una probeta $50 \mathrm{~mL}$ de bebida $\left(\mathrm{V}_{0}\right)$ y luego de 24 horas, en reposo, registrar el volumen que aún se mantiene en emulsión $\left(\mathrm{V}_{s}\right)$ (zona por debajo de la zona clarificada). Con estos datos se aplicó la ecuación (3):

Donde:

$$
\text { Suspensión }(\%)=\frac{V s}{V o} \times 100
$$

$\mathrm{V}_{\mathrm{s}}=$ Volumen en suspensión $(\mathrm{mL})$

$\mathrm{V}_{\mathrm{o}}=$ Volumen inicial de bebida pasteurizada $(\mathrm{mL})$

La variable RPM consideró a los microorganismos: bacterias aerobias totales, coliformes totales, E. coli, mohos y levaduras. La ecuación (4) permitió conocer la reducción porcentual de cada uno. Para determinar el contaje total por placa se utilizó el procedimiento descrito en el acápite 2.2.

Donde:

$$
R P M=100-\left(\frac{C T_{B P}}{C T_{B N P}} * 100\right)
$$

RPM = Reducción de la población microbiana (\%)

$\mathrm{CT}_{\mathrm{BP}}=$ Contaje total en placa para la bebida pasteurizada

$\mathrm{CT}_{\mathrm{BNP}}=$ Contaje total en placa para la bebida no pasteurizada

Las tres variables de salida: VPS ${ }_{b}$, porcentaje en suspensión y RPM fueron evaluadas el mismo día luego de la pasteurización. Todas las mediciones fueron realizadas a temperatura ambiente.

\subsection{Caracterización nutricional del producto obtenido}

Mediante un análisis proximal se determinó la cantidad de sólidos (diferencia de humedad), proteína, extracto etéreo, cenizas y fibra cruda. Los métodos de cuantificación para cada componente son los mismos del apartado 2.2.

\subsection{Análisis estadístico}

Los datos experimentales fueron evaluados en el software STATGRAPHICS Centurion XV.II, se utilizó el análisis de varianza (ANOVA) con un nivel de confianza del $95 \%$. Para hallar las condiciones de solubilización de proteína se aplicó un test LSD. Un análisis de optimización de múltiples respuestas (menor VPS $_{\mathrm{b}}$ y mayor porcentaje en suspensión) determinó los parámetros de elaboración de la bebida a escala piloto.

\section{Resultados y discusión}

\subsection{Caracterización de la harina de quinua}

El análisis granulométrico de la Tabla 3, muestra que el $91 \%$ de partículas posee un tamaño < $212 \mu \mathrm{m}$. Acorde con la norma INEN 3042 referente a "Harina de quinua. Requisitos", el porcentaje de partículas que deben atravesar el tamiz de abertura $212 \mu \mathrm{m}$ es del $95 \%$. El incumplimiento de la norma podría estar asociado al molino de pines utilizado en la molienda, que se clasifica como un equipo de partículas intermedias-finas (Lee \& Henthorn, 2012).

A base del análisis proximal de la Tabla 4 y el análisis microbiológico de la Tabla 5 se afirma que la quinua cumple con los requisitos bromatológicos y microbiológicos establecidos en Ecuador, Bolivia y Perú para el grano. Un análisis proximal de valores similares fue reportado por Tapia et al. (2016) para quinua variedad INIAP Tunkahuan. Incluso, el contenido de proteína de la harina (14.62 $\pm 0,29 \mathrm{~g} / 100 \mathrm{~g})$ es cercano al valor 
promedio (14.2 g/100 g) descrito por Reyes-Montaño et al. (2006) para variedades de quinua en Ecuador.

Tabla 3. Análisis granulométrico de la harina de quinua

\begin{tabular}{|c|r|r|}
\hline $\begin{array}{l}\text { Tamiz norma ASTM } \\
\text { E-11 }\end{array}$ & $\begin{array}{l}\text { Abertura } \\
(\boldsymbol{\mu m})\end{array}$ & $\begin{array}{l}\text { Retenido } \\
(\mathbf{g} / \mathbf{1 0 0} \mathbf{~ g})\end{array}$ \\
\hline 70 & 212 & 9.00 \\
\hline 80 & 180 & 20.40 \\
\hline 100 & 150 & 56.00 \\
\hline 200 & 75 & 14.20 \\
\hline 230 & 63 & 0.20 \\
\hline Fondo & --- & 0.20 \\
\hline
\end{tabular}

Tabla 4. Análisis proximal de la harina de quinua

\begin{tabular}{|l|l|l|l|}
\hline Análisis & $\begin{array}{l}\text { Harina de quinua } \\
\text { Tunkahuan* } \\
\mathbf{( g / 1 0 0 ~ g ) ~}\end{array}$ & $\begin{array}{l}\text { Harina de quinua } \\
\text { INIAP Tunkahuan } \\
(\mathbf{g} / \mathbf{1 0 0} \mathbf{~ g})\end{array}$ & $\begin{array}{l}\text { Harina de quinua } \\
\text { INEN 3042 } \\
\mathbf{( g / * *} / \mathbf{1 0 0} \mathbf{~ g})\end{array}$ \\
\hline Humedad & $9.52 \pm 0.15$ & $11.09 \pm 0.31$ & $13.50(\mathrm{max})$ \\
\hline Proteína & $14.62 \pm 0.29$ & $14.15 \pm 0.28$ & $10.00(\mathrm{~min})$ \\
\hline Extracto etéreo & $6.92 \pm 0.04$ & $6.73 \pm 0.15$ & $4.00(\mathrm{~min})$ \\
\hline Cenizas & $2.61 \pm 0.00$ & $2.58 \pm 0.02$ & $3.00(\mathrm{max})$ \\
\hline Fibra cruda & $2.79 \pm 0.49$ & $3.90 \pm 0.22$ & $1.70(\mathrm{~min})$ \\
\hline $\begin{array}{l}\text { Carbohidratos } \\
\text { totales }\end{array}$ & $66.34 \pm 0.10$ & -- \\
\hline
\end{tabular}

Fuentes: ${ }^{* *}$ Tapia el at., 2016; ${ }^{* \star *}$ INEN 3042, 2015

Tabla 5. Análisis microbiológico de la harina de quinua

\begin{tabular}{|l|l|l|l|}
\hline Microorganismo & $\begin{array}{l}\text { Resultado } \\
(\mathbf{U F C} / \mathbf{m L})\end{array}$ & $\begin{array}{l}\text { Máximo } \\
\text { permisible } \\
(\mathrm{UFC} / \mathbf{g} \text { o } \mathbf{~ m L})\end{array}$ & Cumplimiento \\
\hline B. Aerobias totales & $5.6 \mathrm{E}+04$ & $3 \mathrm{E}+05^{*}$ & Cumple \\
\hline Coliformes totales & 120 & $1 \mathrm{E}+03^{*}$ & Cumple \\
\hline E. coli & 65 & $1 \mathrm{E}+02^{* *}$ & Cumple \\
\hline Mohos y levaduras & $1.05 \mathrm{E}+03$ & $1 \mathrm{E}+04^{* *}$ & Cumple \\
\hline
\end{tabular}

Fuentes: *NB NA 0038, 2007; **NTP 205.062, 2009; ** INEN 3042, 2015

\subsection{Condiciones de solubilización de proteína de quinua a escala laboratorio}

Luego de analizar las variables independientes harina-solución de $\mathrm{NaOH}$, temperatura de acondicionamiento y $\mathrm{pH}$ de acondicionamiento durante 30 minutos en el extracto proteico, se encontró que $\mathrm{VPS}_{\mathrm{e}}$ no incrementó significativamente, y al contrario, en la mayoría de tratamientos la concentración de PS disminuyó con el transcurso del tiempo. Por tanto, se decidió no considerar el tiempo de acondicionamiento (30 min) en los procesos posteriores y continuar el análisis de este objetivo con las concentraciones de PS obtenidas al tiempo 0 min de acondicionamiento.

Las concentraciones de PS al tiempo 0 min de acondicionamiento se observan en la Tabla 6. Los porcentajes varían entre 63 al $77 \%$. Estudios previos con diversas metodologías han logrado solubilizar entre el 10 al $70 \%$ de proteína (Bergesse et al., 2015; Elsohaimy et al., 2015; Föste et al., 2015; Pereira, 2011). 
Tabla 6. Proteína soluble del extracto proteico de quinua a 0 min de acondicionamiento

\begin{tabular}{|c|c|c|c|c|}
\hline \multirow{3}{*}{$\begin{array}{l}\mathrm{T} \\
\left({ }^{\circ} \mathrm{C}\right)\end{array}$} & \multirow{3}{*}{ pH } & \multicolumn{3}{|c|}{ Relación Harina-Solución de $\mathrm{NaOH}$ (w: w) } \\
\hline & & $1: 2.50$ & $1: 3.25$ & $1: 4.00$ \\
\hline & & \multicolumn{3}{|c|}{ Proteína soluble (\%) } \\
\hline \multirow{2}{*}{20} & 7.50 & $67.48 \pm 5.48^{\mathrm{abcd}}$ & $77.10 \pm 1.76^{e}$ & $65.77 \pm 4.07 \mathrm{abc}$ \\
\hline & 8.00 & $72.27 \pm 9.30^{\text {bcde }}$ & $67.54 \pm 1.73^{\mathrm{abcd}}$ & $74.10 \pm 7.88^{\mathrm{de}}$ \\
\hline \multirow{2}{*}{45} & 7.50 & $65.18 \pm 3.86^{\mathrm{ab}}$ & $64.58 \pm 2.70^{a}$ & $63.60 \pm 0.82^{a}$ \\
\hline & 8.00 & $77.09 \pm 1.21^{\mathrm{e}}$ & $72.65 \pm 0.81^{\text {cde }}$ & $68.81 \pm 0.65^{\mathrm{abcc}}$ \\
\hline
\end{tabular}

Letras diferentes indican diferencias significativas entre los tratamientos

$\mathrm{T}$ : Temperatura de acondicionamiento del extracto proteico $\mathrm{pH}: \mathrm{pH}$ de acondicionamiento del extracto proteico

Acorde con el análisis de varianza (ANOVA), el pH fue la única variable que tuvo un efecto significativo $(p<0,05)$, de orden positivo, sobre la concentración de PS en el extracto proteico a 0 min de acondicionamiento. Elsohaymi et al. (2015), Föste et al. (2015) y Ogungbenle (2003) mencionan la influencia del pH en la PS de quinua e indican que entre $6>\mathrm{pH}<10$, la concentración de PS incrementa proporcionalmente en función del pH. En este rango existe mayor solubilidad proteica debido a que soluciones alcalinas provocan la ionización de los grupos carboxilo y la desprotonación de los grupos amino, de tal manera que la proteína se carga negativamente y con ello se crean más interacciones hidrófilas. Asimismo, la repulsión generada por cargas del mismo signo favorece y mantiene la interacción de las proteínas en el medio (Valenzuela et al., 2013). Sin embargo, un pH muy alcalino tampoco es favorable; por lo que según Callisaya \& Alvarado (2009) es recomendable mantener la proteína a un $\mathrm{pH}<9$, de esta manera existe una cantidad apreciable del macronutriente en el medio sin alterar demasiado su conformación química.

De la Tabla 6, se entiende que los tratamientos a condiciones 1:3.25 harina-solución de $\mathrm{NaOH}, 20{ }^{\circ} \mathrm{C}, \mathrm{pH}$ 7,50 y 1:4.00 harina-solución de $\mathrm{NaOH}, 45 \stackrel{\circ}{\circ} \mathrm{C}, \mathrm{pH} 8$ son estadísticamente iguales ( $\mathrm{p}<0.05)$; pero, se escogió el tratamiento a $20{ }^{\circ} \mathrm{C}$ con $77.10 \pm$ $1.76 \%(770.95 \pm 17.57 \mathrm{mg} / \mathrm{mL})$ de PS pues a pH 7.50 la tonalidad del extracto proteico se asemejaba más a las bebidas vegetales comerciales (colores tierra).

\subsection{Condiciones de procesamiento de la bebida de quinua pasteurizada a escala piloto}

A escala piloto, se evaluaron las variables de salida: $V_{P S}$, porcentaje en suspensión y RPM al día 0 a temperatura ambiente. Los resultados de las dos primeras variables se exponen en la Tabla 7.

Tabla 7. Variación de proteína soluble y porcentaje en suspensión al día 0 para bebidas de quinua pasteurizadas

\begin{tabular}{|l|l|l|l|}
\hline $\begin{array}{l}\mathbf{T} \\
\left({ }^{\circ} \mathbf{C}\right)\end{array}$ & $\begin{array}{l}\text { Estabilizante } \\
(\%)\end{array}$ & $\begin{array}{l}\text { VPS } \\
(\%)\end{array}$ & $\begin{array}{l}\text { Porcentaje en suspensión } \\
(\%)\end{array}$ \\
\hline \multirow{3}{*}{120} & 0.00 & $-4.13 \pm 0.72^{\mathrm{a}}$ & $78.67 \pm 3.06^{\mathrm{b}}$ \\
\cline { 2 - 4 } & 0.25 & $-2.36 \pm 0.25^{\mathrm{b}}$ & $98.33 \pm 1.53^{\mathrm{c}}$ \\
\hline \multirow{2}{*}{135} & 0.00 & $-1.83 \pm 0.20^{\mathrm{b}}$ & $69.00 \pm 2.65^{\mathrm{a}}$ \\
\cline { 2 - 4 } & 0.25 & $-3.82 \pm 0.18^{\mathrm{a}}$ & $96.00 \pm 4.00^{\mathrm{c}}$ \\
\hline
\end{tabular}

$\bar{x} \pm \sigma(n=3)$

Letras diferentes indican diferencias significativas entre los tratamientos por columnas

$\mathrm{T}$ : Temperatura de pasteurización

VPS : Variación de proteína soluble al día 0 para la bebida 
La VPS besultó ser negativa en todos los ensayos, lo cual indica que la concentración de PS se reduce luego del tratamiento térmico. Además, se observa que el porcentaje de estabilizante en la bebida provoca cambios significativos $(p<0.05)$ sobre el porcentaje en suspensión. La RPM no varió significativamente, pues se logró eliminar entre el $98 \%$ al $100 \%$ de la microbiota inicial (datos no presentados).

Los cambios en la concentración de PS así como los bajos porcentajes de suspensión en bebidas sin estabilizante $(0.00 \%)$ se deben posiblemente a la temperatura de pasteurización. Rustom et al. (1996) manifiestan que la severidad del tratamiento térmico afecta negativamente a la solubilidad proteica y al porcentaje en suspensión en bebidas de maní pasteurizadas a $137^{\circ} \mathrm{C}$ por 4 y $20 \mathrm{~s}$; situación similar a la hallada. En relación con la estabilidad de suspensiones, Ferragut et al. (2011) mencionan que al pasteurizar las bebidas vegetales, la suspensión se desestabiliza y las partículas sedimentables tienden a separarse de la fase continua. Ya que el extracto utilizado en la bebida es rico en proteínas, parte de esas partículas sedimentables posiblemente puede ser proteína insoluble producto de la desnaturalización por efecto del calor (Rustom et al., 1996), la otra fracción de sedimentables correspondería a los componentes de los aditivos alimenticios. La desnaturalización proteica provoca la coagulación de las proteínas y un aumento de la superficie hidrofóbica (Thuresson, 2015).

Por su parte, la presencia de estabilizante $(0.25 \%)$ mejora notablemente el porcentaje en suspensión $(p<0.05)$ independientemente del tratamiento térmico empleado. En la industria de alimentos, los estabilizantes a base de hidrocoloides reducen la sedimentación de partículas porque favorecen el aumento de la viscosidad de la fase continua, evitan la sinéresis y son más estables a los cambios térmicos (Guerrero, 2010; Obsidián, 2016). El efecto positivo del estabilizante es explicado por Thuresson (2015), según el autor, se pueden añadir agentes externos que estabilicen la suspensión de bebidas vegetales cuando se ha removido algún componente propio del alimento.

EI análisis ANOVA demostró que la interacción entre la temperatura de pasteurización y estabilizante por una parte ejerce una influencia negativa sobre la VPS $\mathrm{b}_{\mathrm{b}} \mathrm{y}$ a su vez tiene un efecto positivo sobre el porcentaje en suspensión $(p<0.05)$.

A través de la optimización de múltiples respuestas para incrementar la VPS V $_{b}$ porcentaje en suspensión se encontraron las mejores condiciones de operación de la bebida a escala piloto: $120 \stackrel{\circ}{ } \mathrm{C}$ y $0.25 \%$ de estabilizante. Según la norma guatemalteca COGUANUR NTG 34031 para leche de soya, tomada como referencia , la pasteurización UHT se define como un proceso a temperaturas entre los 120 y $144{ }^{\circ} \mathrm{C}$ por períodos mínimos de $2 \mathrm{~s}$ (Ministerio de Salud Pública y Asistencia Social, 2005). En este estudio, el tiempo de residencia fue de $1 \mathrm{~s}$ a $120^{\circ} \mathrm{C}$; por consiguiente, al no cumplir con la definición, el tratamiento aplicado sería pseudo UHT.

\subsection{Caracterización nutricional del producto obtenido}

En el análisis proximal de la bebida de quinua, Tabla 8 , se observa que luego del procesamiento el contenido de macronutrientes es menor en comparación con la harina de quinua. El extracto proteico constituyó la fase líquida en el proceso de extracción y por lo tanto contenía el agua de disolución de $\mathrm{NaOH}$ utilizada. Este hecho fue un causal que se tenga un menor contenido de sólidos y por lo tanto de macronutrientes en la bebida de quinua.

Una disminución en el contenido de carbohidratos, fibra y proteína se encuentra asociada también a una pérdida de estos macronutrientes durante la primera etapa de elaboración; esto acorde con la patente de Scanlin \& Stone (2009), pues en este documento se explica que el subproducto de la solubilización alcalina de la proteína de quinua es una torta constituida por carbohidratos, fibra y proteína insoluble. Además, es probable que la torta haya arrastrado consigo otros materiales que no se solubilizaron a $\mathrm{pH}$ alcalino. 
Tabla 8. Análisis proximal de la bebida de quinua

\begin{tabular}{|l|l|}
\hline Análisis & $\begin{array}{l}\text { Bebida de quinua pasteurizada } \\
\mathbf{( g / 1 0 0 ~} \mathbf{~})\end{array}$ \\
\hline Sólidos & $13.35 \pm 0.01$ \\
\hline Proteína & $2.69 \pm 0.00$ \\
\hline Extracto etéreo & $1.34 \pm 0.04$ \\
\hline Cenizas & $0.39 \pm 0.01$ \\
\hline Fibra cruda & $0.05 \pm 0.04$ \\
\hline Carbohidratos totales & $8.93 \pm 0.05$ \\
\hline \multicolumn{2}{|c|}{$\overline{\mathrm{x}} \pm \sigma(\mathrm{n}=2)$} \\
\hline
\end{tabular}

La torta, desechada luego de la centrifugación, tenía una fase de color blanco, debido a esto es posible decir que la fracción de carbohidratos eliminada correspondió a almidón de quinua. En consecuencia, la porción de carbohidratos $(8.93 \pm 0.05 \mathrm{~g} / 100 \mathrm{~g})$ en la bebida podrían ser azúcares (monosacáridos, disacáridos o pentosanos) provenientes del grano (Valencia-Chamorro, 2003) y los aditivos alimenticios agregados en la formulación. Sin embargo, también pudieron estar presentes restos de almidón que no lograron separarse por completo.

La bebida de quinua en este estudio logró superar en contenido proteico $(2.69 \pm 0.00$ $\mathrm{g} / 100 \mathrm{~g}$ ) a otras bebidas comerciales de arroz, coco, macadamia, avellana, avena y almendra que contienen entre 0.07 y $2.11 \mathrm{~g} / 100 \mathrm{~g}$ de este nutriente. No obstante, aún no supera a las bebidas de soya que contienen $3,70 \mathrm{~g} / 100 \mathrm{~g}$ de proteína (Jeske et al., 2017).

Rubico et al. (1987) reportó que una bebida de maní pasteurizada a $121 \stackrel{\circ}{\circ} \mathrm{C}$ durante 3 s tiene $13.72 \%$ de sólidos totales y $2.56 \%$ de proteína ( $2.56 \%$ ); valores similares a los mostrados en la Tabla 8 para los mismos componentes.

\section{Conclusiones y recomendaciones}

El aumento de $\mathrm{pH}$ favoreció la solubilidad proteica no así la temperatura y el tiempo de acondicionamiento.

La temperatura de pasteurización $120{ }^{\circ} \mathrm{C}$ y la adición de $0.25 \%$ de estabilizante fueron las mejores condiciones para la obtención de una bebida a base de quinua. Los tratamientos térmicos disminuyeron la solubilidad proteica y desestabilizaron las partículas, esta última se atenuó con la adición de un estabilizante en la formulación de la bebida.

Se obtuvo una bebida con 2.69 \% de proteína de quinua con calidad microbiológica aceptable al día 0 que cumple con los parámetros exigidos en bebidas similares a base de vegetales.

Las condiciones óptimas (harina-solución de $\mathrm{NaOH}$, temperatura y $\mathrm{pH}$ de extracción) para obtener PS de quinua, determinadas en esta investigación podrían ser consideradas para aislar o concentrar proteína de quinua. Los parámetros determinados a escala piloto (temperatura de pasteurización y porcentaje de estabilizante) servirían de base para optimizar procesos industriales. Se sugiere realizar un análisis PDCAAS, un estudio de digestibilidad in vitro y un análisis de estabilidad de la bebida para determinar el tiempo de vida útil.

\section{Bibliografía}

3M. (2003). Guía de Interpretación Petrifilm 3M. Recuperado de http://jornades.uab.cat/workshopmrama/sites/jornades.uab.cat.workshopmrama/files /Petrifilm_guias.pdf

AOAC. (2005). Official methods of analysis of AOAC international: Current Trough Revision 2, 2007. (18 ${ }^{\text {th }}$. ed.). Virginia, USA.

Bergesse, A. E., Boiocchi, P. N., Calandri, E. L., Cervilla, N. S., Gianna, V., Guzmán, C. A., Miranda, P., Montoya, P. \& Mufari, J. R. (2015). Aprovechamiento Integral del Grano 
de Quinoa. Aspectos Tecnológicos, Fisicoquímicos, Nutricionales y Sensoriales. (1era. ed.). Córdoba, Argentina: s/e. Recuperado de https://rdu.unc.edu.ar/bitstream/handle/11086/1846/Aprovechamiento\%20integral\% 20del\%20grano\%20de\%20quinoa.pdf?sequence=7\&isAllowed=y

Callisaya, C., \& Alvarado, A. (2009). Aislados Proteínicos De Granos Altoandinos Chenopodiaceas; Quinua "Chenopodium Quinoa" - Cañahua "Chenopodium Pallidicaule" por Precipitación Isoeléctrica. Revista Boliviana de Química, 26(1), 12 20. Recuperado de http://www.scielo.org.bo/pdf/rbq/v26n1/v26n1a02.pdf

Chito, D., Ortega, R., Ahumada, A. \& Rosero, B. (2017). Quinoa (Chenopodium quinoa Willd.) versus soja (Glycine max [L] Merr) en la nutrición humana: revisión sobre las características agroecológicas, de composición y tecnológicas. Revista Española de Nutrición Humana y Dietética, 21(2), 184-198. http://doi.org/10.14306 /renhyd.21.2.256

Elsohaimy, S. A., Refaay, T. M., \& Zaytoun, M. A. M. (2015). Physicochemical and functional properties of quinoa protein isolate. Annals of Agricultural Sciences, 60(2), 297-305. http://doi.org/10.1016/j.aoas.2015.10.007

FAO. (2011). La quinua: cultivo milenario para contribuir a la seguridad alimentaria mundial. Córdoba: FAO. Recuperado de http://www.fao.org/fileadmin/templates/aiq2013 /res/es/cultivo_quinua_es.pdf

Ferragut, V., Hernández-Herrero, M., Poliseli, F., Valencia, D., \& Guamis, B. (2011). Ultra High Pressure Homogenization (UHPH) treatment of vegetable milks: improving hygienic and colloidal stability. En Proceedings of the 11th International Congress on Engineering and Food (ICEF11) - Food Process Engineering in a Changing World (Vol. 2, pp. 1193-1198). Recuperado de http://www.icef11.org/content /papers/fms/fms480.pdf

Föste, M., Elgeti, D., Brunner, A., Jekle, M., \& Becker, T. (2015). Food and Bioproducts Processing Isolation of quinoa protein by milling fractionation and solvent extraction. Food and Bioproducts Processing, 96, 20-26. http://doi.org/10.1016/j.fbp .2015.06.003

Guerrero, A. (2010). Elaboración de leche de avena esterilizada utilizando diferentes estabilizantes (gelatina y obsigel) y niveles de Pimalac como conservante en la empresa Prolac S.E.M. Escuela Superior Politécnica de Chimborazo. Recuperado de http://dspace.espoch.edu.ec/bitstream/123456789/810/1/27T0168.pdf

Hariyadi, P. (2013). Hot-Fill Processing of Beverages. Food Reviews International, 1(1), 46-49. Recuperado de https://www.researchgate.net/publication/259255039_HOTFILL PROCESSING OF BEVERAGES

ICC. (1972). International Association for Cereal Science and Technology. Determination of crude fibre value. Quality Assurance and Safety of Crops and Foods, 8 (3), 319480. Recuperado de https://www.icc.or.at/standard methods/113

Ismail, M. (2015). Which Is Better for Humans, Animal Milk or Vegetable Milk. J Nutr Health Food Eng, 2(5), 14-15. http://doi.org/10.15406/jnhfe.2015.02.00067

Jacobsen, S., Mujica, A., \& Ortiz, R. (2003). La importancia de los cultivos andinos. Fermentum. Revista Venezolana de Sociología Y Antropología, 13(36), 14-24. Recuperado de http://www.redalyc.org/html/705/70503603/

Jeske, S., Zannini, E., \& Arendt, E. K. (2017). Evaluation of Physicochemical and Glycaemic Properties of Commercial Plant-Based Milk Substitutes. Plant Foods for Human Nutrition, 72(1), 26-33. http://doi.org/10.1007/s11130-016-0583-0

Kaur, I., \& Tanwar, B. (2016). Quinoa Beverages: Formulation, Processing and Potential Health Benefits. Rom J Diabetes Nutr Metab Dis., 23(2), 215-225. http://doi.org/10.1515/rjdnmd-2016-0026

Kwok, K.-C., \& Niranjan, K. (1995). Review: Effect of thermal processing on soymilk. International Journal of Food Science \& Technology, 30(3), 263-295. http://doi.org/10.1111/j.1365-2621.1995.tb01377.x 
Lee, S., \& Henthorn, K. (2012). Particle Technology and Applications. (1era. ed.). USA: Taylor \& Francis Group.

Mäkinen, O. (2014). Studies on quinoa (Chenopodium quinoa) for novel food and beverage applications. University College Cork, Irlanda. Recuperado de https://cora.ucc.ie/bitstream/handle/10468/2018/141205_e-ThesisOutiMakinen.pdf ?sequence $=2 \&$ isAllowed $=\mathrm{y}$

Mäkinen, O. E., Uniacke-Lowe, T., O'Mahony, J. A., \& Arendt, E. K. (2015). Physicochemical and acid gelation properties of commercial UHT-treated plant-based milk substitutes and lactose free bovine milk. Food Chemistry, 168, 630-638. http://doi.org/10.1016/j.foodchem.2014.07.036

Mäkinen, O.E., Wanhalinna, V., Zannini, E., \& Arendt, E. K. (2015). Foods for Special Dietary Needs: Non-Dairy Plant Based Milk Substitudes. Critical Reviews in Food Sciencie and Nutricion, 56(3), 339-349. http://doi.org/10.1080/10408398. 2012.761950

Ministerio de Salúd Pública y Asistencia Social. (2005). Reglamento técnico de soya natural fluída. Recuperado de http://www.puntofocal.gov.ar/notific_otros_miembros /gtm60_t.pdf

NB NA 0038. (2007). Granos andinos-Pseudo cereales-Quinua en grano-Clasificación y Requisitos. Recuperado de http://www.conal.gob.ar/CONASE/actas /ActaCONASE_2013_04Abr18_Anexol.pdf

NTE INEN 517. (1980). Harinas de origen vegetal. Determinación del tamaño de las partículas. Recuperado de https://law.resource.org/pub/ec/ibr/ec.nte.0517.1981.pdf

NTE INEN 3042. (2015). Harina de quinua. Requisitos. Recuperado de http://www.normalizacion.gob.ec/wp-content/uploads/downloads/2016/01 /nte_inen_3042.pdf

NTP 205.062. (2009). Quinua (Chenopodium quinoa Willd). Requisitos. Recuperado de http://www.conal.gob.ar/CONASE/actas/ActaCONASE_2013_04Abr18_Anexoll.pdf

Obsidián. (2016). Bebidas. Recuperado de http://obsidian.com.ec/alimentos/bebidas/

Oficina Comercial de Ecuador en Los Ángeles, E. U., \& Dirección de Inteligencia Comercial e Inversiones. (2011). Perfil de quinua y elaborados en Estados Unidos. Pro Ecuador. Recuperado de http://www.proecuador.gob.ec/wp-content/uploads/2015/02 /PROECU_PPM2011_QUINUA_ESTADOS-UNIDOS.pdf

Ogungbenle, $\mathrm{H}^{-}$N. (2003). Nutritional evaluation and functional properties of quinoa (Chenopodium quinoa) flour. International Journal of Food Sciences and Nutrition, 54(2), 153-158. http://doi.org/10.1080/0963748031000084106

Pereira, S. (2011). Elaboración de leche de quinua (Chenopodium quinoa, Willd). Escuela Politécnica Nacional, Quito, Ecuador. Recuperado de http://bibdigital.epn.edu.ec /bitstream/15000/2646/1/CD-3329.pdf

Pineli, L. D. L. D. O., Botelho, R. B. A., Zandonadi, R. P., Solorzano, J. L., Oliveira, G. T. De, Reis, C. E. G., \& Teixeira, D. S. (2015). Low glycemic index and increased protein content in a novel quinoa milk. Food Science and Technology, 63(2), 1261-1267. http://doi.org/10.1016/j.Iwt.2015.03.094

Pro Ecuador. (2015). Análisis sectorial: Quinua. Quito: Pro Ecuador. Recuperado de http://www.proecuador.gob.ec/wp-content/uploads/2015/10/PROEC_AS2015 QUINUA.pdf

Reyes-Montaño, E. A., Ávila-Torres, D. P., \& Guevara-Pulido, J. O. (2006). Componente nutricional de diferentes variedades de quinua de la región andina. AVANCES Investigación En Ingeniería, (5), 86-97. Recuperado de http://www.unilibre.edu.co $/$ revistaavances/avances-5/r5_art10.pdf

Rubico, S. M., Resurrección, A. V. A., Frank, J. F., \& Beuchat, L. R. (1987). Suspension Stability, Texture, and Color of High Temperature Treated Peanut Beverage. Journal of Food Science, 52(6), 1676-1679. http://doi.org/10.1111/j.13652621.1987.tb05904.x 
Rustom, I. Y. S., López-Leiva, M. H., \& Nair, B. M. (1996). Nutritional, sensory and physicochemical properties of peanut beverage sterilized under two different UHT conditions. Food Chemistry, 56(1), 45-53. http://doi.org/10.1016/03088146(95)00153-0

Scanlin, L., \& Stone, M. (2009). Quinoa Protein Concentrate, Production and Functionality. Estados Unidos. Recuperado de https://docs.google.com/viewer?url=patentimages .storage.googleapis.com/pdfs/US7563473.pdf

Sethi, S., Tyagi, S. K., \& Anurag, R. K. (2016). Plant-based milk alternatives an emerging segment of functional beverages: a review. Journal of Food Science and Technology, 53(9), 3408-3423. http://doi.org/10.1007/s13197-016-2328-3

Soteras, E. M. (2011). Obtención y formulación de una bebida en base de granos de amaranto. Universidad Nacional del Litoral, Santa Fé, Argentina. Recuperado de http://bibliotecavirtual.unl.edu.ar:8080/tesis/bitstream/handle/11185/342/tesis.pdf?se quence $=1$

Tapia, I. L., Taco, D. R., \& Taco, V. J. (2016). Aislamiento de proteínas de quinua ecuatoriana ( Chenopodium quinoa Willd ) variedad INIAP Tunkahuan con remoción de compuestos fenólicos , para uso potencial en la nutrición y salud humanas. Revista de La Facultad de Ciencias Médicas (Quito), 41(1), 71-80. Recuperado de http://revistadigital.uce.edu.ec/index.php/CIENCIAS_MEDICAS/article/view/249/299

Thuresson, C. (2015). Development and studies on a gluten free, liquid suspension based on quinoa (Chenopodium quinoa). Swedish University of Agricultural Sciences, Suecia. Recuperado de https://stud.epsilon.slu.se/8665/7/thuresson_c_151203.pdf

Trevino, S. R., Scholtz, J. M., \& Pace, C. N. (2008). Measuring and Increasing Protein Solubility. Journal of Pharmaceutical Sciences, 97(10), 4155-4166. http://doi.org/10.1002/jps.21327

Valencia-Chamorro, S. A. (2003). Quinoa. En: Caballero B.: Encyclopedia of Food Science and Nutrition. Vol. 8. Academic Press, Amsterdam: 4895-4902.

Valencia, E., Cuéllar, I., Aguilar, I., Salmerón, A., \& Rodríguez, F. (2012). Implementación del método de biuret para la determinación de proteína residual en lactosuero. Revista Sistemas Ambientales, 5(1), 17-20. Recuperado de https://es.scribd.com/document/329452857/Extenso-de-Biuret-Caseina

Valenzuela, C., Abugoch, L., Tapia, C., \& Gamboa, A. (2013). Effect of alkaline extraction on the structure of the protein of quinoa (Chenopodium quinoa Willd.) and its influence on film formation. International Journal of Food Science and Technology, 48(4), 843849. http://doi.org/10.1111/ijfs.12035

Villacrés, E., Peralta, E., Egas, L., \& Mazón, N. (2011). Potencial Agroindustrial de la Quinua. Quito: INIAP. Recuperado de http://quinua.pe/wpcontent/uploads/2014/02/Potencial-Agroindustrial-de-la-quinua-1.pdf

Zayas, J. (1997). Solubility of Proteins. En Functionality of Proteins in Food (1 ed., pp. 675). Berlin, Heidelbeg: Springer. https://doi.org/10.1007/978-3-642-59116-7_2 